\title{
The role of taxanes in triple-negative breast cancer: literature review [Corrigendum]
}

Mustacchi G, De Laurentiis M. Drug Des Devel Ther. 2015;9:4303-4318.

On page 4315, Disclosure section, "This work was supported by an unconditional grant from Editree editors, Italy, and both authors received a fee. Giorgio Mustacchi receives honoraria from Roche, Celgene, and EISAI. Michelino De Laurentiis receives honoraria from Roche, Celgene, EISAI, Novartis, and AstraZeneca. The authors report no other conflicts of interest in this work." should have read "The authors report no conflict of interest with this work". 